\title{
Primary intrinsic defects and their charge transition levels in $\beta-\mathrm{Ga}_{2} \mathrm{O}_{3}$
}

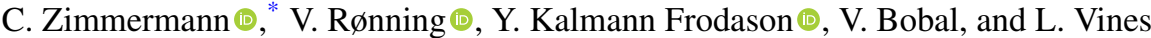 \\ Physics Department/Centre for Materials Science and Nanotechnology, University of Oslo, P. O. Box 1048, Blindern, Oslo N-0316, Norway \\ J. B. Varley $\odot$ \\ Lawrence Livermore National Laboratory, Livermore, California 94550, USA
}

(Received 10 December 2019; revised 30 April 2020; accepted 23 June 2020; published 24 July 2020)

\begin{abstract}
A steady-state photocapacitance (SSPC) setup directly connected to the beamline of a MeV ion implanter is utilized to study primary intrinsic defects in $\beta-\mathrm{Ga}_{2} \mathrm{O}_{3}$ generated by $\mathrm{He}$ implantation at cryogenic temperatures $(120 \mathrm{~K})$. At low temperatures, the migration of defects is suppressed, and hence the generation of primary intrinsic defects is expected to prevail. SSPC measurements reveal defect-related optical transitions in halide vapor-phase epitaxy (HVPE) -grown $\beta-\mathrm{Ga}_{2} \mathrm{O}_{3}$ thin films with onset energies at $1.3\left(T_{1}\right), 1.7\left(T_{2}\right), 1.9\left(T_{3}\right), 2.6$ $\left(T_{4}\right), 3.7\left(T_{5}\right)$, and $4.2 \mathrm{eV}\left(T_{6}\right) . T_{2}, T_{4}, T_{5}$, and $T_{6}$ were observed in as-received HVPE-grown $\beta$ - $\mathrm{Ga}_{2} \mathrm{O}_{3}$ thin films, whereby $T_{2}$ is only sporadically observed. The introduction rates for $T_{3}, T_{4}$, as well as $T_{6}$ indicate an origin related to primary intrinsic defects. Notably, $T_{1}$ and $T_{3}$ are only observed after He implantation at cryogenic temperatures. Hybrid-functional calculations were performed to estimate the optical absorption cross-section spectra for the gallium $\left(\mathrm{Ga}_{\mathrm{i}}\right)$ and oxygen $\left(\mathrm{O}_{\mathrm{i}}\right)$ interstitials as well as the corresponding vacancies ( $V_{\mathrm{Ga}}$ and $V_{\mathrm{O}}$, respectively), and compared with the measured onsets for optical absorption found by SSPC measurements. Indeed, we propose $T_{3}$ to be associated with $\mathrm{Ga}_{\mathrm{i}}^{(+/+2)}$ and/or $V_{\mathrm{GaI}}^{(-3 /-2)}$, while $T_{4}$ is suggested to be related to $V_{\mathrm{O} K}^{(0 /+)}(K=\mathrm{I}, \mathrm{II}$, III $)$ and/or $V_{\mathrm{GaII}}^{(-3 /-2)}$. Additionally, several further charge-state transition levels associated with $V_{\mathrm{GaI}}$ and $V_{\mathrm{GaII}}$ may contribute to $T_{4}$ and $T_{6}$. We further studied the kinetics of the defects created with He implantation by exposing the sample to room temperature. The kinetics observed for $T_{3}$ and $T_{4}$ further support the proposed assignments of the corresponding defect signatures.
\end{abstract}

DOI: 10.1103/PhysRevMaterials.4.074605

\section{INTRODUCTION}

Beta gallium oxide $\left(\mathrm{Ga}_{2} \mathrm{O}_{3}\right)$ is a wide-band-gap semiconductor exhibiting exceptionally high breakdown electrical fields [1], and hence it has attracted considerable attention in recent years as a potential candidate for applications in UV sensors and power electronics [1-4]. Intrinsic as well as extrinsic defects have a profound influence on the optical and electrical properties of the material. For example, defects can act as recombination centers limiting the efficiency of UV sensors, or as traps for charge carriers and affect the performance of devices for power electronics [4-7]. Thus, understanding prominent defects in $\mathrm{Ga}_{2} \mathrm{O}_{3}$ is of the utmost importance in enabling the material to live up to its potential.

First-principles defect calculations predict several electronic states within the band gap of $\mathrm{Ga}_{2} \mathrm{O}_{3}$ associated with the primary intrinsic defects [8-19]. Gallium vacancies $\left(V_{\mathrm{Ga}}\right)$ are expected to be deep acceptors, the gallium interstitial $\left(\mathrm{Ga}_{\mathrm{i}}\right)$ is associated with donor states, the oxygen vacancies $\left(V_{\mathrm{O}}\right)$ give rise to deep donor states, and the oxygen interstitial $\left(\mathrm{O}_{\mathrm{i}}\right)$ can be either a donor or an acceptor depending on its configuration [15-17,19]. Notably, $V_{\mathrm{O}}$ and $V_{\mathrm{Ga}}$ can occur in different configurations [15-17,19].

Experimentally, a multitude of electronic states within the band gap of $\mathrm{Ga}_{2} \mathrm{O}_{3}$ have so far been unveiled using deep-level

*christian.zimmermann@fys.uio.no transient spectroscopy (DLTS) [15,20-26], deep-level optical spectroscopy (DLOS) [26-28], steady-state photocapacitance (SSPC) measurements [22,23,25-30], as well as a variety of other methods [23-25,29,31-35]. For example, SSPC studies performed by Farzana et al. as well as Zhang et al. on $\mathrm{Ga}_{2} \mathrm{O}_{3}$ reveal electronic states inside the $\mathrm{Ga}_{2} \mathrm{O}_{3}$ band gap at around $2.2,3.2$, and $4.4 \mathrm{eV}$ below the conduction-band edge $\left(E_{\mathrm{C}}\right)$ [26-28], while at least six defect levels have been identified in the upper part of the band gap by DLTS [15,20,21,24-26]. Identifying the microscopic origin of the observed defect signatures, however, remains challenging, and hence only a few assignments have been made so far. One notable exception is the case of $\mathrm{Fe}_{\mathrm{Ga}}$, which was shown to introduce an electronic state at around $0.78 \mathrm{eV}$ below $E_{\mathrm{C}}$ and identified by DLTS [21]. The electronic levels associated with intrinsic defects and their complexes, however, have so far escaped experimental identification.

One approach to studying intrinsic defects in semiconductors is by intentionally introducing such defects via irradiation or implantation using neutrons, protons, $\alpha$-particles, electrons, or heavier ions [15,23,28,36,37]. $\mathrm{Ga}_{\mathrm{i}}$ and $\mathrm{O}_{\mathrm{i}}$ are, however, expected to be very mobile at, or even below, room temperature $[14,15,38]$. Furthermore, one can expect impurities, like hydrogen, to have a low migration barrier, and hence they are likely to passivate or form complexes with (intrinsic) defects. Notably, it has been implied by simulations as well as experimental studies that, for example, hydrogen and $V_{\mathrm{Ga}}$ can form complexes [19,39]. 


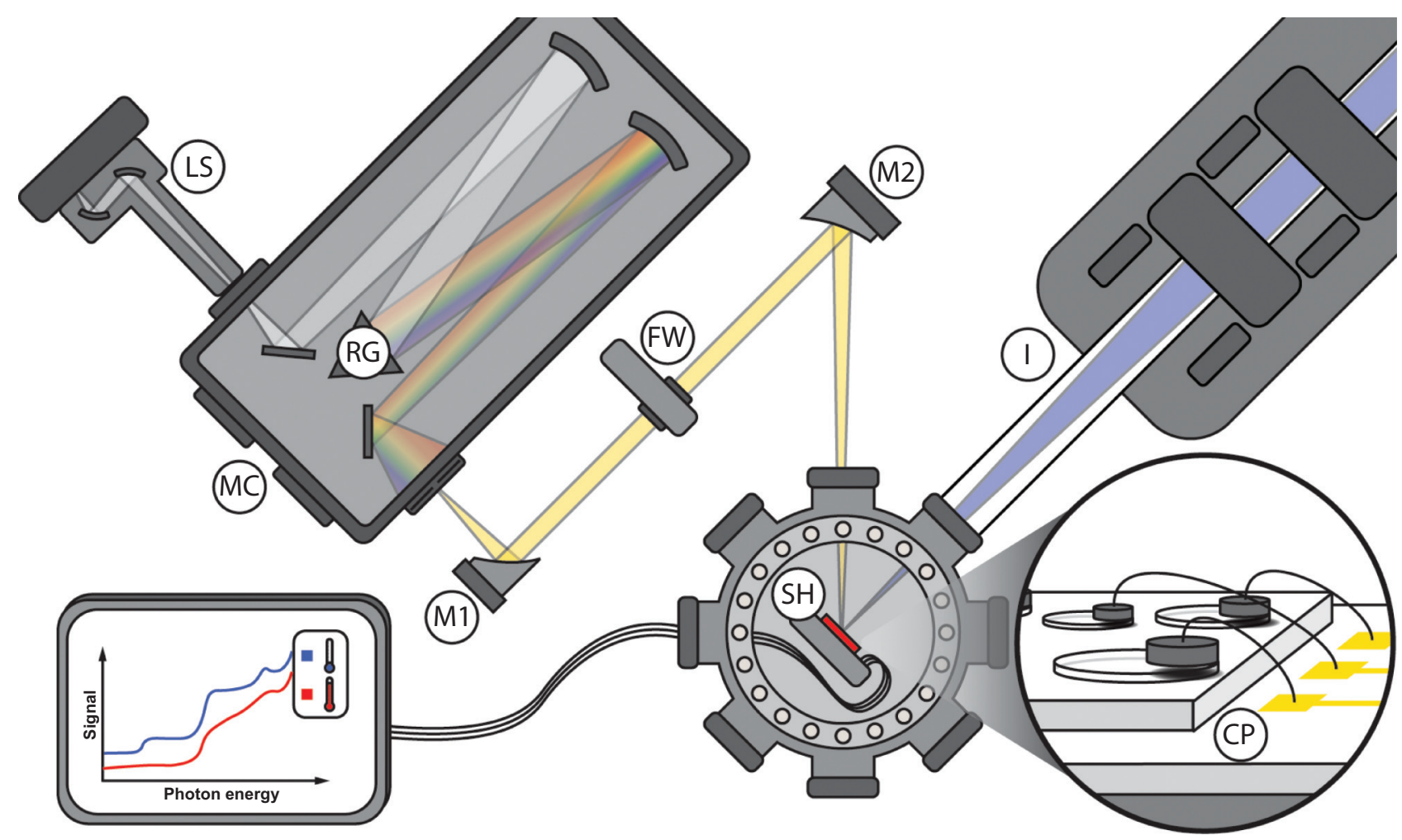

FIG. 1. Overview of the setup used for on-line SSPC measurements. The optical excitation at variable wavelength is provided by a white light source (LS) dispersed by a monochromator (MC) utilizing a grating (RG). The near-monochromatic light is steered toward the sample (SH) using parabolic mirrors (M1 and M2). Long-pass filters (FW) are used to suppress artifacts due to second-order diffraction. The sample (SH) is placed inside a cryostat that is directly connected to the beam line of a MeV ion implanter (I), and hence SSPC measurements can be performed on-line. The inset shows the contact layout: Semitransparent contacts are electrically connected by wire-bonding to minimize shadowing for light as well as for ions.

At sufficiently low temperatures, however, primary intrinsic defects as well as impurities are immobile, and hence the formation of primary intrinsic defects should prevail during low-temperature irradiation or implantation. This can be utilized by combining low-temperature irradiation or implantation with characterization at the beamline of an accelerator (on-line), i.e., irradiation at cryogenic temperatures and subsequent characterization without heating up the sample $[40,41]$. Moreover, subsequent exposure of the sample to higher temperatures can shed light on the defect kinetics in the corresponding temperature range, potentially revealing defect complex formation or passivation of defects.

In this work, we present results from on-line SSPC measurements on $\mathrm{Ga}_{2} \mathrm{O}_{3}$ thin films grown by halide vapor-phase epitaxy implanted with $\mathrm{He}$ at $120 \mathrm{~K}$. Our measurements reveal several new defect signatures that we propose to be associated with primary intrinsic defects. In particular, $\mathrm{Ga}_{\mathrm{i}}$ and/or $V_{\mathrm{Ga}}$ formed on a tetrahedral Ga site $\left(V_{\mathrm{GaI}}\right)$ are potential candidates for a level with an onset for optical absorption at around $1.9 \mathrm{eV}$. We further propose that $V_{\mathrm{O}^{-}}$ and other $V_{\mathrm{Ga}}$-related defects show optical absorption in the region of 2.5-3.7 eV. These results are further corroborated by hybrid-functional calculations estimating the optical absorption expected for $\mathrm{Ga}_{\mathrm{i}}$ and $V_{\mathrm{Ga}}$ and $V_{\mathrm{O}}$. Additionally, our results indicate that passivation and/or migration of such defects indeed occurs already at temperatures below room temperature.

\section{METHODOLOGY}

\section{A. Experimental details}

The $\mathrm{Ga}_{2} \mathrm{O}_{3}$ thin films studied here were grown by halide vapor-phase epitaxy (HVPE) on conductive $\mathrm{Ga}_{2} \mathrm{O}_{3}$ substrates and obtained from Novel Crystal Technology, Inc. [4]. The thin films are unintentionally doped with $\mathrm{Si}$ and exhibit nominal charge-carrier concentrations in the range of $5 \times 10^{16}-3 \times$ $10^{17} \mathrm{~cm}^{-3}$. The thickness of the thin films is around $10 \mu \mathrm{m}$, while their surface corresponds to the (001) plane. Using a laser cutter, the as-obtained wafers were cut into pieces measuring approximately $5 \times 5 \mathrm{~mm}^{2}$. The sample pieces were subsequently cleaned with acetone, isopropanol, and deionized water for $5 \mathrm{~min}$ each inside an ultrasonic bath. Afterward, Ohmic backside contacts consisting of Ti (thickness $=10 \mathrm{~nm}$ ) and $\mathrm{Al}$ (thickness $=150 \mathrm{~nm}$ ) were deposited by e-beam evaporation. After a subsequent cleaning step, semitransparent $\mathrm{Ni}$ (thickness $=20 \mathrm{~nm}$ ) Schottky contacts were deposited by e-beam evaporation using a shadow mask with contact diameters of 300, 600, and $900 \mu \mathrm{m}$. To ensure mechanical stability of the Ni contacts for wire-bonding, a second deposition of $\mathrm{Ni}$ (thickness $=150 \mathrm{~nm}$ ) was performed using a shadow mask (with only circular openings displaying a diameter of $300 \mu \mathrm{m}$ ), which aligned with the semitransparent Ni contacts. The contact layout is shown in the inset of Fig. 1. All results shown here were obtained on semitransparent contacts with a diameter of $600 \mu \mathrm{m}$. Wire-bonding on the thick part of the 
$\mathrm{Ni}$ contact was used for connecting the sample to electrical measurement instruments.

$\mathrm{He}$ ions were implanted at $120 \mathrm{~K}$ with an energy of $180 \mathrm{keV}$ and fluences $\Phi$ ranging from $1.25 \times 10^{11}$ to $5 \times 10^{11} \mathrm{~cm}^{-2}$. Negligible heating of the sample during $\mathrm{He}$ implantation is expected due to the utilization of low ion currents as well as low ion fluences. The defect generation was simulated utilizing Monte Carlo simulations implemented within the The Stopping and Range of Ions in Matter (SRIM) package [42] using displacement energies for $\mathrm{Ga}$ and $\mathrm{O}$ of 25 and $28 \mathrm{eV}$, respectively. Simulations were run for $\mathrm{Ni}$ thicknesses of $20 \mathrm{~nm}$ (semitransparent region) and $170 \mathrm{~nm}$ (thick $\mathrm{Ni}$ region used for wire-bonding), resulting in projected ranges $R_{\mathrm{p}}$ of $400 \mathrm{~nm}$ for a Ni thickness of $170 \mathrm{~nm}$, and $610 \mathrm{~nm}$ for a Ni thickness of $20 \mathrm{~nm}$, respectively. Notably, during He implantation primary intrinsic defects are generated regardless of their formation energy in thermal equilibrium [42]. After generation, these primary intrinsic defects may form energetically more favorable configurations if they are mobile enough at the sample temperature to relax into the corresponding configurations.

Current-voltage (IV) and capacitance-voltage (CV) measurements were carried out under dark conditions at room temperature as well as at $120 \mathrm{~K}$ using a Keithley 6487 picoammeter/voltage source and a Boonton 7200 capacitance meter, respectively. $\mathrm{CV}$ measurements were performed at a probing frequency $f_{\text {probe }}$ of $1 \mathrm{MHz}$. A relative static dielectric constant $\varepsilon_{\mathrm{S}}$ of 10.2 was assumed for $\mathrm{Ga}_{2} \mathrm{O}_{3}$ [43]. CV and IV measurements were performed inside a closed-cycle He cryostat connected to the beamline of an $\mathrm{MeV}$ ion implanter. From the forward bias region of IV curves, we extracted the ideality factor of the investigated Schottky junctions in order to verify the suitability of the junctions for SSPC measurements [44].

SSPC measurements were conducted utilizing the same closed-cycle $\mathrm{He}$ cryostat connected to the beamline of an $\mathrm{MeV}$ ion implanter (on-line) as was used for IV and CV measurements. Figure 1 shows the layout of the experimental setup used for on-line SSPC measurements. For studying defect kinetics, subsequent heat treatments up to room temperature were performed inside the same cryostat without transferring the sample. During SSPC measurements, the capacitance of the junction is recorded after illuminating the junction at a certain wavelength $\lambda$ or photon energy $E$ for a given time. A constant angle of incidence was used for illumination. Here, the photocapacitance was recorded after 5 min using a Boonton 7200 capacitance meter $\left(f_{\text {probe }}=1 \mathrm{MHz}\right)$ while the junction was kept at a fixed external bias (typically between -8 and $-1 \mathrm{~V})$. A laser-driven light source (EQ-77 from Energetiq) was used as a white light source, covering the spectral range from 190 to $2500 \mathrm{~nm}$. The unpolarized light was dispersed with a grating-based monochromator (Shamrock 500i from ANDOR). Utilizing a grating with 1200 lines $/ \mathrm{mm}$ (blaze at $400 \mathrm{~nm}$ ) as well as fully opened exit and entrance slits at $2.5 \mathrm{~mm}$, a typical spectral resolution of $\sim 20 \mathrm{meV}$ was obtained. Long-pass filters were used to ensure that no light from second-order diffraction reaches the sample. Typical photon fluxes of $1 \times 10^{17} \mathrm{~m}^{-2} \mathrm{~s}^{-1}$ in the UV and $1 \times 10^{19} \mathrm{~m}^{-2} \mathrm{~s}^{-1}$ in the visible part of the spectrum were estimated using a calibrated thermal power meter placed at the sample position inside the cryostat. The near-monochromatic light was deliv- ered to the sample using parabolic mirrors. The sample was illuminated starting at the long-wavelength end (usually at around $1600 \mathrm{~nm}$ ), proceeding to shorter wavelengths in steps of 20 or $40 \mathrm{meV}$. In contrast to other studies, no filling pulse or above-band-gap illumination was utilized during SSPC measurements (see, for example, Refs. [28,44]). Samples were, however, preilluminated for $30 \mathrm{~min}$ at the longest wavelength used for a specific measurement range. This ensures that at the beginning of the measurement, traps responding to longer wavelengths than the ones used for the SSPC measurement are being ionized, and hence the sample is in or close to a steady state for illumination at such wavelengths. For some samples, the preillumination is not sufficient to reach comparable conditions as were encountered for measurement ranges involving longer wavelengths. For these samples, small discontinuities are seen close to the spectral positions where long-pass filter changes occurred. These discontinuities can be regarded as experimental artifacts.

The resulting photocapacitance signal is accumulative and represents the persistent charge created within the depletion region of the Schottky diode upon illumination at a certain photon energy. SSPC spectra recorded using different measurement ranges usually displayed slight offsets compared to each other. Offsets caused by switching between different measurement ranges were corrected assuming them to be independent of photon energy and using the measurement range for the lowest photon energies as reference.

SSPC spectra $S(E)$ are usually presented as

$$
S(E)=2 \frac{C_{\text {illumination }}(E)-C_{\text {dark }}}{C_{\text {dark }}} N_{\mathrm{D}}=2 \frac{\Delta C_{\text {illumination }}(E)}{C_{\text {dark }}} N_{\mathrm{D}} .
$$

Here, $E$ is the photon energy and $C_{\text {illumination }}(E)$ denotes the (photo)capacitance due to illumination at a certain photon energy $E . C_{\text {dark }}$ represents the capacitance measured at a fixed reverse bias prior to illumination, and hence $\Delta C_{\text {illumination }}(E)$ denotes the change in capacitance due to illumination. $N_{\mathrm{D}}$ represents the donor concentration. Each electronic state inside the band gap will give rise to a steplike feature in SSPC spectra, and hence SSPC spectra can be represented by the following empirically motivated model:

$$
S(E)=2 N_{\mathrm{D}} \sum_{i} \frac{\Delta C_{\text {illumination, } \mathrm{i}}}{C_{\mathrm{dark}}} \frac{1}{1+\exp \left(-\frac{E-E_{\mathrm{i}}}{\gamma_{\mathrm{i}}}\right)},
$$

where the individual contributions $i$ are described by sigmoidal functions. $\Delta C_{\text {illumination, } \mathrm{i}} / C_{\mathrm{dark}}$ represents the relative change in capacitance due to illumination for an individual contribution and is independent of $E$. For a specific contribution $i, E_{\mathrm{i}}$ describes the position of the onset of the SSPC signal and $\gamma$ represents the steepness of the corresponding step. Features with $\Delta C_{\text {illumination,i }} / C_{\text {dark }}>0$ (optically induced electron emission) as well as $\Delta C_{\text {illumination,i }} / C_{\text {dark }}<0$ (optically induced hole emission) can occur [26-28,44,45]. SSPC spectra can also be represented as derivatives with respect to photon energy $[45,46]$, where steplike features are transformed into peaklike features. Such a representation can greatly improve the interpretation of the technique, and aid deconvolution [46,47].

The height $2 N_{\mathrm{D}} \Delta C_{\text {illumination, } \mathrm{i}} / C_{\mathrm{dark}}$ of each step is related to the effective trap concentration $N_{\mathrm{t}, \mathrm{i}}^{\text {eff }}$ and serves as a lower 
bound for the actual concentration $N_{\mathrm{t}, \mathrm{i}}$ [45]. For small total trap concentrations $N_{\mathrm{t}}$ or $\Delta C_{\text {illumination }} / C_{\text {dark }} \ll 0.5$, a linear relationship holds between $\Delta C_{\text {illumination, } \mathrm{i}} / C_{\text {dark }}$ and $N_{\mathrm{t}, \mathrm{i}}^{\text {eff }}$ :

$$
N_{\mathrm{t}, \mathrm{i}}^{\mathrm{eff}}=2 \frac{\Delta C_{\text {illumination, }}}{C_{\text {dark }}} N_{\mathrm{D}} \frac{W^{2}}{W^{2}-x_{1}^{2}} .
$$

Here, $W$ is the depletion layer width. $x_{1}$ denotes the depth where the electronic state of the trap level associated with contribution $i$ crosses the Fermi level $E_{\mathrm{F}}$. For determining $x_{1}$, one needs to know the thermodynamic charge transition level for a specific defect. This level can be estimated from $E_{\mathrm{i}}$ [see Eq. (2)] by assuming a value for the Franck-Condon shift $d_{\mathrm{FC}}[48,49]$. The term $W^{2} /\left(W^{2}-x_{1}^{2}\right)$ is often called $\lambda$-correction [44].

For larger total trap concentrations $N_{\mathrm{t}}$ or $\Delta C_{\text {illumination }} / C_{\text {dark }} \approx 0.5$, Eq. (3) does not hold and the trap concentrations can be estimated from $\Delta C_{\text {illumination,i }} / C_{\text {dark }}$ by numerically solving the steady-state capacitance under illumination for $N_{\mathrm{t}, \mathrm{i}}^{\mathrm{eff}}$. More information about how to extract $N_{\mathrm{t}, \mathrm{i}}^{\text {eff }}$ in the case of low and large total trap concentrations can be found in the Supplemental Material [50].

Notably, all defects inside the space-charge region with a thermodynamic charge-state transition level below the Fermi level will contribute to the measured SSPC signal $S(E)$. Moreover, the value determined for $N_{\mathrm{t}, \mathrm{i}}^{\text {eff }}$ will depend on the competition between optically induced hole and electron emission for the trap i $[44,45]$ as well as the mobility of electrons and holes (see the Supplemental Material [50]).

\section{B. Computational details}

First-principles calculations were performed using the projector augmented wave method (PAW) [51,52] and the Heyd-Scuseria-Ernzerhof screened hybrid functional (HSE) [53], as implemented in the VASP code [54]. The fraction of screened Hartree-Fock exchange was adjusted to $\alpha=0.32$, which accurately describes both the experimental band gap $\left(E_{\mathrm{g}}=4.9 \mathrm{eV}\right)$ and structural parameters as reported elsewhere $[21,55,56]$. The experimentally determined band-gap value can be expected to exhibit an uncertainty of around $\pm 0.1 \mathrm{eV}$ [55]. The Ga $3 d$ and Ti 3p, 3d, as well as $4 s$ electrons were included as valence states. For defect calculations, we used 160atom supercells, a plane-wave energy cutoff of $400 \mathrm{eV}$, and a single special $k$-point at $(1 / 4,1 / 4,1 / 4)$. Defect formation energies and thermodynamic charge-state transition levels were calculated by following the well-established formalism [57]. For charged defects, we adopted the anisotropic [58] Freysoldt, Neugebauer, and Van de Walle scheme to correct formation energies [59], and the method recently proposed by Gake et al. to correct vertical transition energies [60]. Optical absorption energies of defects were estimated by using the effective one-dimensional configuration coordinate (CC) model with parameters obtained from the HSE calculations [49,59]. However, the absorption onset will be lower than the classical absorption energy $E_{\text {abs }}$ obtained from CC diagrams due to vibrational broadening. Absorption cross sections that include vibrational broadening can be simulated based on $\mathrm{CC}$ diagrams by following the scheme outlined in Refs. [49,61].

Following Refs. [49,61], defect-related optical absorption is modeled as an electronic transition between a ground state (defect in charge state $q$ and charge carrier localized at or close to the defect) and an excited state (defect in charge state $q \pm 1$ and delocalized charge carrier in the conduction or valence band) triggered by a photon. Despite the complex optical selection rules in $\mathrm{Ga}_{2} \mathrm{O}_{3}$ due to the crystal symmetry and orbital character of the conduction and valence bands [55,56,62,63], the corresponding optical transitions are assumed to be allowed. Notably, defect-related optical transitions can be expected to be allowed if the transition is accompanied by strong lattice relaxation [64], which has been reported for various defects in $\mathrm{Ga}_{2} \mathrm{O}_{3}$ [19]. Moreover, the defect potential is assumed to be represented by a $\delta$-function, in accordance with what is typically assumed for deep-level defects $[61,65]$. The excited state is a continuum of states in the conduction or valence band, whereby the bands are assumed to be parabolic. We note that we focus on transitions to the conduction band around $E_{\mathrm{C}}$, which is a highly dispersive state that is predominantly parabolic and exhibits a simpler orbital character than the upper valence bands [55,56,62,63]. For the determination of the onset of defect-related optical absorption, the parabolic approximation is not believed to introduce a large error. Furthermore, the transition matrix element relevant for the electronic transition is assumed to be constant, i.e., to be independent of energy or momentum. The model proposed by Alkauskas et al. [49] and Kopylov et al. [61] takes phonon contributions into account: (i) phonons are emitted during the absorption process, and (ii) ground and excited states possess vibrational substates that will be occupied at higher temperatures and contribute to the optical absorption. It is assumed that the phonon contributions can be modeled by using an effective frequency for the phonons relevant for the ground and excited state, respectively. Notably, both (i) and (ii) will contribute to a broadening of the defect-related optical absorption cross-section spectra and a corresponding shift of the absorption onset. Phonon emission (i) will contribute to the broadening already at $0 \mathrm{~K}$, whereas the contribution of additional vibrational substates (ii) will become more pronounced with increasing temperature. The calculations presented in this work were performed at $0 \mathrm{~K}$. For a selection of defects, optical absorption cross-section spectra were also calculated assuming $120 \mathrm{~K}$, and we found only minor differences. More information regarding the model used for calculating defect-related optical absorption cross-section spectra and their temperature dependence can be found in the Supplemental Material [50].

Migration barriers were evaluated using the climbing nudged elastic band method (cNEB) [66], using at least five images and requiring the resulting force to be $\leqslant 0.03 \mathrm{eV}^{-1}$. Due to their large computational cost, barriers were evaluated using the same supercell geometries and the PBEsol functional [67] and PAW potentials that treated the Ga $3 d$ electrons in the core. The lowest barriers were evaluated with HSE using the same approach to assess the differences.

\section{RESULTS}

\section{A. Computational results}

Hybrid-functional calculations were performed to predict optical signatures originating from various primary intrinsic 
(a)

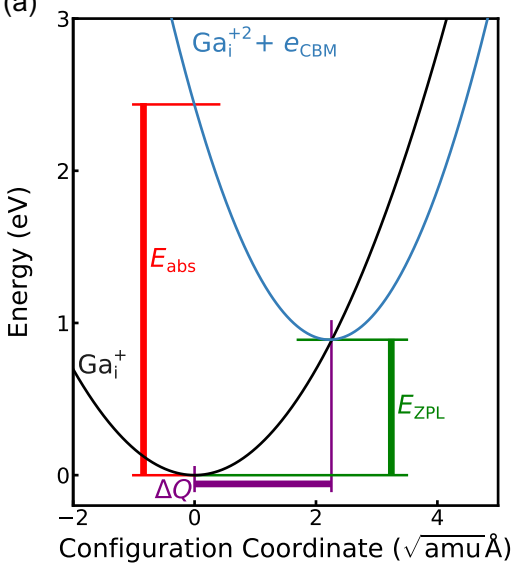

(b)

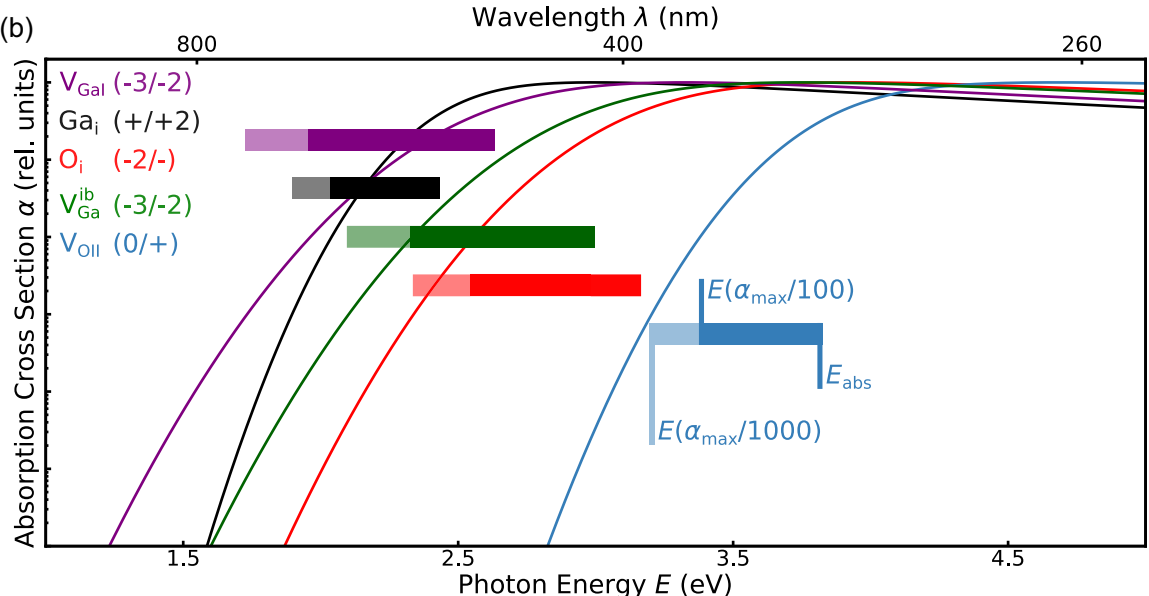

FIG. 2. (a) CC diagram for the $(+/+2)$ charge-state transition of $\mathrm{Ga}_{\mathrm{i}}$. The vertical transition energy connected to optical absorption is marked as $E_{\mathrm{abs}}$, while the thermodynamic transition level is marked as $E_{\mathrm{ZPL}}$. The minima of the parabola signify the (meta)stable configurations of $\mathrm{Ga}_{i}^{+}$and $\mathrm{Ga}_{\mathrm{i}}^{+2}$, respectively. The corresponding difference in the configuration coordinate $\Delta Q$ is also marked in the plot. The values for $E_{\mathrm{abs}}, E_{\mathrm{ZPL}}$, and $\Delta Q$ for $\mathrm{Ga}_{\mathrm{i}}^{(+/+2)}$ as well as various other primary intrinsic defects and some defect complexes involving intrinsic defects are summarized in Table I. (b) Absorption cross-section spectra calculated for charge-state transitions of various primary intrinsic defects. The bars represent the interval defined by $E_{\text {abs }}$ [as defined in (a)] and $E\left(\alpha_{\max } / 1000\right)$ for the corresponding transition. The position of $E\left(\alpha_{\max } / 100\right)$ is also indicated. $E\left(\alpha_{\max } / 1000\right)$ marks the photon energy at which the absorption cross section decreased by three orders of magnitude as compared to $\alpha_{\max }$, whereas $E\left(\alpha_{\max } / 100\right)$ marks the photon energy at which the absorption cross section decreased by two orders of magnitude as compared to $\alpha_{\max }$.

defects in $\mathrm{Ga}_{2} \mathrm{O}_{3}$. Specifically, we computed $\mathrm{CC}$ diagrams to obtain optical absorption spectra related to charge-state transitions associated with the primary intrinsic defects. For each defect, we focused on the charge-state transition with the energetically lowest transition energy associated with optical absorption. However, it can be expected that some primary intrinsic defects will exhibit more than one charge-state transition level inside the band gap of $\mathrm{Ga}_{2} \mathrm{O}_{3}$ [15]. Moreover, we only considered charge-state transitions involving the defects and the conduction band (optically induced electron emission). Due to the likely formation of self-trapped holes in $\mathrm{Ga}_{2} \mathrm{O}_{3}[68,69]$, we assume transitions between the defects and the valence band to not significantly contribute to the measured SSPC spectra (see the Supplemental Material [50]). Calculations were performed for $\mathrm{Ga}_{\mathrm{i}}, \mathrm{O}_{\mathrm{i}}, V_{\mathrm{OK}}(K=\mathrm{I}$, II, III), and $V_{\mathrm{Ga} J}(J=\mathrm{I}, \mathrm{II}) . V_{\mathrm{Ga} J}(J=\mathrm{I}, \mathrm{II})$ was shown previously to prefer significantly relaxed configurations labeled as $V_{\mathrm{Ga}}^{\mathrm{i} M}$ $(M=a, c, b)$ [15]. The energy barrier for $V_{\mathrm{GaJ}}(J=\mathrm{I}, \mathrm{II})$ to transform into $V_{\mathrm{Ga}}^{\mathrm{i} M}(M=a, c, b)$ is predicted to be around $0.5-0.7 \mathrm{eV}[15,19]$. Optical signatures related to absorption were also calculated for $V_{\mathrm{Ga}}^{\mathrm{i} M}(M=a, c, b) . V_{\mathrm{OK}}(K=\mathrm{I}, \mathrm{II}, \mathrm{III})$, $V_{\mathrm{Ga} J}(J=\mathrm{I}, \mathrm{II})$, and $V_{\mathrm{Ga}}^{\mathrm{i} M}(M=a, c, b)$ are defined according to Refs. $[15,16]$. $V_{\mathrm{OI}}$ and $V_{\mathrm{OII}}$ have a threefold symmetry, while $V_{\mathrm{OIII}}$ exhibits a fourfold symmetry. $V_{\text {GaI }}$ is a gallium vacancy on a tetrahedral gallium site, while $V_{\text {GaII }}$ denotes a gallium vacancy on an octahedral site. $V_{\mathrm{Ga}}^{\mathrm{i} M}(M=a, b, c)$ are relaxed configurations, essentially consisting of a $\mathrm{Ga}$ atom moving into the gallium vacancy. $V_{\mathrm{Ga}}^{\mathrm{i} M}$ can be viewed as a Ga interstitial with two neighboring Ga half-vacancies [19]. Graphical representations of the calculated structures can be found in the Supplemental Material [50]. Additionally, optical signatures related to absorption were also computed for a selection of favorable defect complexes consisting of $V_{\mathrm{O}}$ and
$V_{\mathrm{Ga}}$ (divacancy complexes) [15]. A thorough study of defect complexes is, however, beyond the scope of this work.

Figure 2(a) shows a CC diagram computed for the chargestate transition from + to +2 for $\mathrm{Ga}_{\mathrm{i}}$. From the $\mathrm{CC}$ diagrams, $E_{\mathrm{abs}}, E_{\mathrm{ZPL}}$, and $\Delta Q$ can be determined. $E_{\mathrm{abs}}$ is the classical absorption energy, while $E_{\mathrm{ZPL}}$ is the energy of the zerophonon line, which describes the thermodynamic charge transition level. $\Delta Q$ is the difference in configuration coordinate between the different charge states of the defect. The parameters $E_{\mathrm{abs}}, E_{\mathrm{ZPL}}$, and $\Delta Q$ are summarized in Table I for various primary intrinsic defects and some divacancy complexes. Typically, the difference between $E_{\mathrm{abs}}$ and $E_{\mathrm{ZPL}}$, and hence the Franck-Condon shift $d_{\mathrm{FC}}$, is in the range of $0.8-1.7 \mathrm{eV}$. This suggests that the charge-state transitions involve a large lattice relaxation. In contrast, significantly lower Franck-Condon shifts were estimated so far experimentally for defects in $\mathrm{Ga}_{2} \mathrm{O}_{3}$ [26-28]. More computational results regarding optical absorption spectra related to primary intrinsic defects can be found in the Supplemental Material [50].

Computed normalized absorption cross-section spectra $(\alpha)$ are shown in Fig. 2(b) for various primary intrinsic defects. An interval was extracted from these spectra covering all photon energies from $E_{\text {abs }}$ [as defined in Fig. 2(a)] to $E\left(\alpha_{\max } / 1000\right)$, while the position of $E\left(\alpha_{\max } / 100\right)$ is also indicated. Hereby, $\alpha_{\max }$ denotes the maximum value of the absorption crosssection spectra. $E\left(\alpha_{\max } / 1000\right)$ is defined as the photon energy where $\alpha$ decreased by three orders of magnitude as compared to $\alpha_{\max }$, whereas $E\left(\alpha_{\max } / 100\right)$ is defined as the photon energy where $\alpha$ decreased by two orders of magnitude as compared to $\alpha_{\max }$. These intervals are used for comparison between computational results and measured SSPC spectra.

To gauge the kinetics of primary intrinsic defects, migration barriers $E_{\mathrm{m}}$ were calculated for $\mathrm{Ga}_{\mathrm{i}}$, while results for 
TABLE I. Summary of parameters for various primary intrinsic defects and some divacancy complexes obtained from hybridfunctional calculations. Calculations were performed for the chargestate transition associated with the energetically lowest transition energy associated with optical absorption. Hereby, only charge-state transitions involving the defects and the conduction band were considered. The parameters $E_{\mathrm{ZPL}}, E_{\mathrm{abs}}$, and $\Delta Q$ are obtained from CC diagrams and defined in Fig. 2(a). $E\left(\alpha_{\max } / 1000\right)$ is defined as the photon energy at which the absorption cross section decreased by three orders of magnitude as compared to $\alpha_{\max }$, whereas $E\left(\alpha_{\max } / 100\right)$ denotes the photon energy at which the absorption cross section decreased by two orders of magnitude as compared to $\alpha_{\max }$.

\begin{tabular}{lcccccc}
\hline \hline & $\begin{array}{c}\text { Charge } \\
\text { state } \\
\text { Defect }\end{array}$ & $\begin{array}{c}\Delta Q \\
\text { transition }\end{array}$ & $\begin{array}{c}E_{\mathrm{ZPL}} \\
(\sqrt{\mathrm{amu}})\end{array}$ & $\begin{array}{c}E_{\mathrm{abs}} \\
(\mathrm{eV})\end{array}$ & $\begin{array}{c}E\left(\frac{\alpha_{\max }}{100}\right) \\
(\mathrm{eV})\end{array}$ & $\begin{array}{c}E\left(\frac{\alpha_{\max }}{1000}\right) \\
(\mathrm{eV})\end{array}$ \\
\hline $\mathrm{Ga}_{\mathrm{i}}$ & $(+/+2)$ & 2.22 & 0.89 & 2.43 & 2.04 & 1.90 \\
$\mathrm{O}_{\mathrm{i}}$ & $(-2 /-)$ & 4.33 & 1.45 & 3.16 & 2.55 & 2.34 \\
$V_{\mathrm{OI}}$ & $(0 /+)$ & 3.85 & 1.68 & 3.20 & 2.79 & 2.63 \\
$V_{\mathrm{OII}}$ & $(0 /+)$ & 3.26 & 2.46 & 3.82 & 3.38 & 3.20 \\
$V_{\mathrm{OIII}}$ & $(0 /+)$ & 3.30 & 1.67 & 3.00 & 2.60 & 2.45 \\
$V_{\mathrm{Ga} I}$ & $(-3 /-2)$ & 2.38 & 1.80 & 2.63 & 1.96 & 1.73 \\
$V_{\mathrm{Ga} I I}$ & $(-3 /-2)$ & 3.12 & 2.35 & 3.21 & 2.62 & 2.40 \\
$V_{\mathrm{Ga}}^{\mathrm{i} a}$ & $(-3 /-2)$ & 2.24 & 2.16 & 3.06 & 2.42 & 2.19 \\
$V_{\mathrm{Ga}}^{\mathrm{i} b}$ & $(-3 /-2)$ & 2.35 & 2.02 & 2.99 & 2.33 & 2.10 \\
$V_{\mathrm{Ga}}^{\mathrm{ic}}$ & $(-3 /-2)$ & 2.37 & 2.65 & 3.48 & 2.82 & 2.59 \\
$V_{\mathrm{Ga}}^{\mathrm{ic}}-V_{\mathrm{OIII}}$ & $(-1 / 0)$ & 2.40 & 3.04 & 4.16 & 3.62 & 3.42 \\
$V_{\mathrm{Ga}}^{\mathrm{i} b}-V_{\mathrm{OI}}$ & $(-1 / 0)$ & 2.32 & 2.41 & 3.61 & 3.06 & 2.85 \\
$V_{\mathrm{GaII}}-V_{\mathrm{OII}}$ & $(-3 /-2)$ & 3.10 & 1.60 & 2.55 & 1.93 & 1.71 \\
\hline \hline
\end{tabular}

$E_{\mathrm{m}}$ are already available for $\mathrm{O}_{\mathrm{i}}, V_{\mathrm{O}}$, and $V_{\mathrm{Ga}}$ in the literature $[14,15,38]$. For $\mathrm{O}_{\mathrm{i}}$, a value of $0.12 \mathrm{eV}$ was calculated for $E_{\mathrm{m}}$ [15]. For $V_{\mathrm{O}}$, values from 1.2 to $4 \mathrm{eV}$ were found, while for $V_{\mathrm{Ga}}$ barriers between 0.5 and $2.3 \mathrm{eV}$ were computed [14]. Notably, the migration of $V_{\mathrm{Ga} J}(J=\mathrm{I}, \mathrm{II})$ is associated with the formation of the configurations $V_{\mathrm{Ga}}^{\mathrm{i} M}(M=a, c, b)$ [19]. However, the barrier for the $V_{\mathrm{Ga}}^{\mathrm{i} M}(M=a, c, b)$ configurations to migrate or transform back into $V_{\mathrm{Ga} J}(J=\mathrm{I}, \mathrm{II})$ are significantly higher [19]. For $\mathrm{Ga}_{\mathrm{i}}$, we find that the lower bound for $E_{\mathrm{m}}$ is strongly dependent on the charge state of $\mathrm{Ga}_{\mathrm{i}}$. We find an $E_{\mathrm{m}}$ of $0.65 \mathrm{eV}$ for $\mathrm{Ga}_{\mathrm{i}}^{+}$, and we compute a barrier of $0.48 \mathrm{eV}$ for $\mathrm{Ga}_{\mathrm{i}}^{3+}$. It is found that $\mathrm{Ga}_{\mathrm{i}}^{2+}$ is metastable and will transform immediately into $\mathrm{Ga}_{i}^{3+}$, meaning $\mathrm{Ga}_{i}$ exhibits so-called negative-U behavior [48]. Larger migration barriers were obtained when performing the calculations using the HSE functional, and hence the migration barriers stated above can be seen as lower bounds. More information regarding these calculations can be found in the Supplemental Material [50].

Following the approach of Kyrtsos et al., a lower temperature can be estimated at which migration of defects will occur [14]. For $V_{\mathrm{O}}$, Kyrtsos et al. estimate that such defects will not be mobile at or below room temperature, while for $V_{\mathrm{Ga}}$ some migration might occur already just below room temperature [14]. Notably, this migration is connected to the transformation of $V_{\mathrm{Ga} J}(J=\mathrm{I}, \mathrm{II})$ into $V_{\mathrm{Ga}}^{\mathrm{i} M}(M=a, c, b)$ [19]. However, the $V_{\mathrm{Ga}}^{\mathrm{i} M}(M=a, c, b)$ configurations are less mobile than $V_{\mathrm{Ga} J}(J=\mathrm{I}$, II) and are not expected to migrate at or below room temperature [19]. For $\mathrm{Ga}_{\mathrm{i}}$, we can estimate that
$\mathrm{Ga}_{i}^{3+}$ will be mobile at around $190 \mathrm{~K}$, while $\mathrm{Ga}_{i}^{+}$will be mobile at around $250 \mathrm{~K} . \mathrm{O}_{\mathrm{i}}$ is expected to be mobile already at around $50 \mathrm{~K}$ given its low migration barrier of $0.12 \mathrm{eV}$ [15]. Note also that electrical fields as they are present within a space-charge region may enhance the diffusion of charged defect species $[14,70]$.

\section{B. Experimental results}

Figure 3(a) shows IV curves recorded on a $\mathrm{Ni} / \mathrm{Ga}_{2} \mathrm{O}_{3} / \mathrm{Ti} / \mathrm{Al}$ junction comprising a HVPE-grown $\mathrm{Ga}_{2} \mathrm{O}_{3}$ thin film. The junction displays a high rectification of around eight orders of magnitude. The investigated junctions generally display rectifications ranging from three to nine orders of magnitude. The series resistances range from several $\Omega$ to a few hundred $\Omega$ depending on the specific junction. The ideality factor at room temperature is typically around 1-1.5, but increases to values of around 3-4 for measurements at $120 \mathrm{~K}$. After He implantation, all diodes exhibit an increase in series resistance, while usually no significant change in the reverse bias region is observed.

In Fig. 3(b), the results of typical CV measurements are displayed for one particular junction [same junction as depicted in Fig. 3(a)]. The donor concentration $N_{\mathrm{D}}$ for all diodes investigated is in the range of $2 \times 10^{16}-2 \times 10^{17} \mathrm{~cm}^{-3}$, whereby the donor profiles generally show a slight decrease in donor concentration toward the surface. We estimate the uncertainty for $N_{\mathrm{D}}$ to be around $10 \%$ due to uncertainties in the contact area and the value of $\varepsilon_{\mathrm{s}}[43,71]$. The typical probing depth is in the range of $300-700 \mathrm{~nm}$, i.e., the main part of the region where implantation-induced defects are to be expected is within the probing volume. The conductance does not exceed $20 \mathrm{mS}$. After He implantation, $N_{\mathrm{D}}$ decreases, resulting in a probing depth of approximately $450-700 \mathrm{~nm}$.

In the following, we will describe the results obtained from SSPC measurements performed at $120 \mathrm{~K}$. Figure 4 displays a SSPC spectrum recorded on an as-received HVPE-grown $\mathrm{Ga}_{2} \mathrm{O}_{3}$ thin film. Steps in the SSPC spectrum can be seen with onset energies at 1.7, 2.6, 3.7, 4.2, and $4.8 \mathrm{eV}$. The corresponding defect signatures are labeled as $T_{2}(1.7 \mathrm{eV}), T_{4}$ $(2.6 \mathrm{eV}), T_{5}(3.7 \mathrm{eV})$, and $T_{6}(4.2 \mathrm{eV})$, while the signature at $4.8 \mathrm{eV}$ is associated with the band gap and is labeled as $E_{\mathrm{g}}$ in Fig. $4 . T_{5}$ is also associated with a decreasing SSPC signal starting at a photon energy of around $3.9 \mathrm{eV}$. The trap concentrations $N_{\mathrm{t}, \mathrm{i}}^{\text {eff }}$ are estimated to be in the range of $1 \times 10^{14} \mathrm{~cm}^{-3}-1 \times 10^{16} \mathrm{~cm}^{-3}$ using Eq. (3). The low defect concentrations confirm that all defect-related photoexcitation processes can be regarded as independent of each other. The detection limit for the sample shown in Fig. 4 is estimated to be around $1 \times 10^{13} \mathrm{~cm}^{-3}$. Notably, SSPC spectra recorded on various other as-received HVPE-grown $\mathrm{Ga}_{2} \mathrm{O}_{3}$ thin films exhibit the same features with the exception of $T_{2}$, which is only sporadically observed.

Figure 4 also displays a comparison between SSPC spectra recorded at $120 \mathrm{~K}$ before and after He implantation performed at $120 \mathrm{~K}$ with a fluence $\Phi$ of $1.25 \times 10^{11} \mathrm{~cm}^{-2}$. Two new signatures with onset energies at around 1.3 and $1.9 \mathrm{eV}$ appear after He implantation. The corresponding signatures were labeled $T_{1}$ and $T_{3}$, respectively. $T_{1}$ does not appear in all samples subjected to He implantation, while $T_{3}$ is detected 

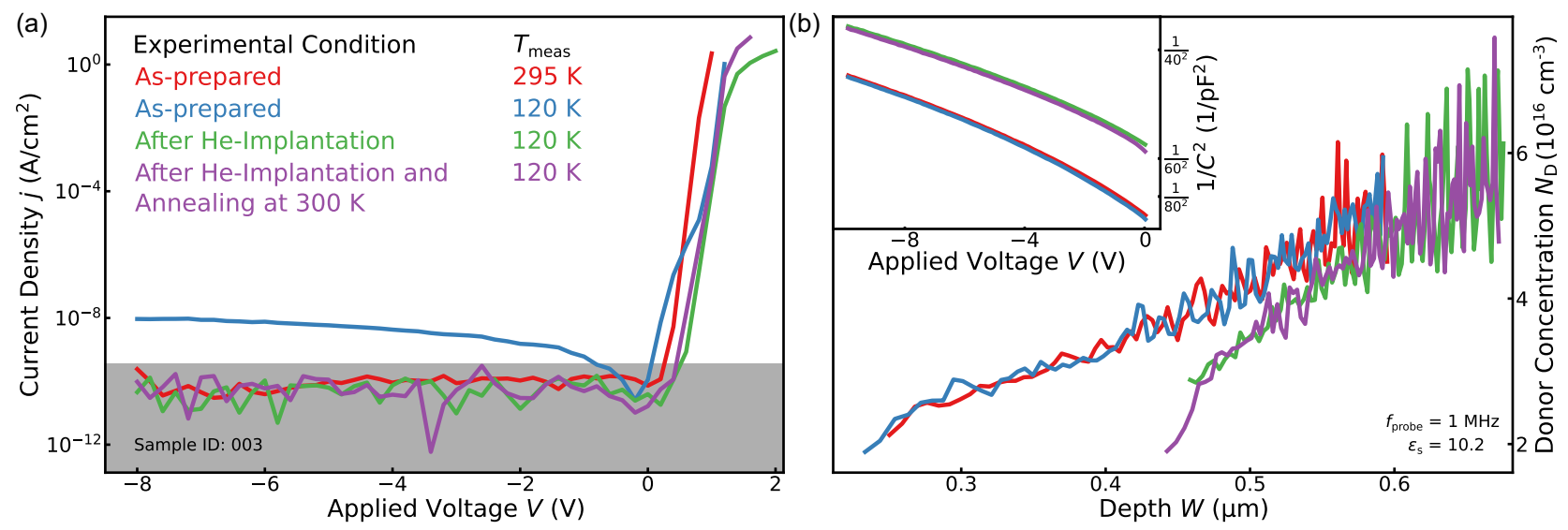

FIG. 3. (a) Results of IV measurements performed on a Ni/Ga $\mathrm{O}_{3} / \mathrm{Ti} / \mathrm{Al}$ junction comprising a $\mathrm{HVPE}$-grown $\mathrm{Ga}_{2} \mathrm{O}_{3}$ thin film. Measurements were conducted at $120 \mathrm{~K}$ and at room temperature for the as-prepared junction. After He implantation at $120 \mathrm{~K}$ as well as after a subsequent heat treatment at room temperature, additional IV measurements were performed. Both these measurements were conducted at $120 \mathrm{~K}$. The junction displays a high rectification of around eight orders of magnitude. The ideality factor is in the range of 1.5-4. No pronounced changes besides an increase in series resistance can be seen in the IV characteristics after He implantation. (b) Results of CV measurements performed on the same junction and for the same experimental conditions as stated in (a). Donor concentration profiles derived from the $\mathrm{CV}$ measurements are displayed. The inset shows $1 / C^{2}$ vs $V$ plots for the same measurements. The donor concentration is in the order of $4 \times 10^{16} \mathrm{~cm}^{-3}$. After He implantation, a decrease in donor concentration can be seen, especially toward the surface of the $\mathrm{Ga}_{2} \mathrm{O}_{3}$ thin film.

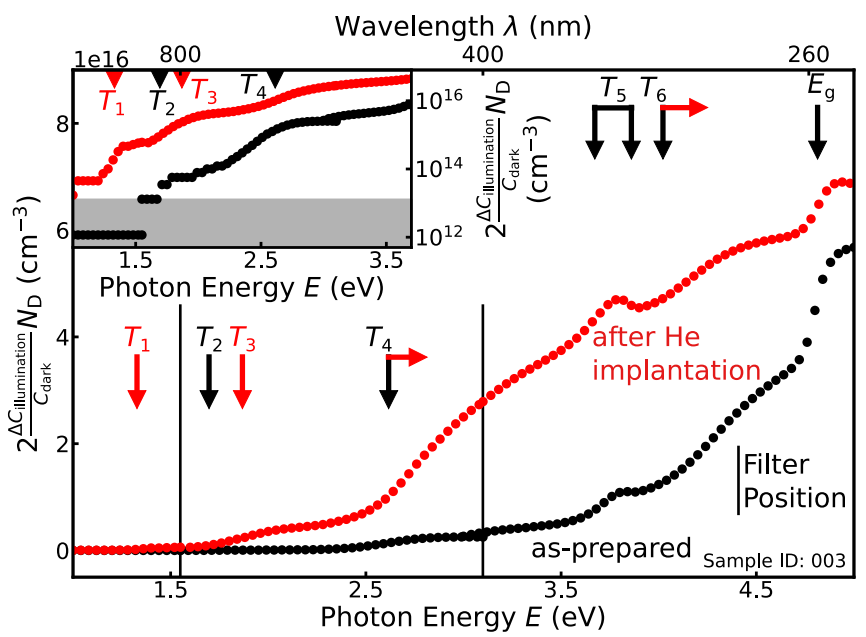

FIG. 4. SSPC spectra recorded on a HVPE-grown $\mathrm{Ga}_{2} \mathrm{O}_{3}$ thin film before and after implantation with $\mathrm{He}$ ions at $120 \mathrm{~K}$. The He ions were implanted with a fluence of $1.25 \times 10^{11} \mathrm{~cm}^{-2}$. The photon energy positions of the low-pass filters are indicated with black vertical lines. Several defect signatures are observed before He implantation and marked with black vertical arrows as well as labeled $T_{2}, T_{4}, T_{5}$, and $T_{6} . T_{5}$ displays an increasing as well as decreasing signal. Furthermore, a signal that can be attributed to the band gap $E_{\mathrm{g}}$ is detected. After He implantation, new features marked with vertical red arrows and labeled $T_{1}$ and $T_{3}$ are observed. The defect signatures $T_{4}$ and $T_{6}$ change in amplitude and exhibit a shift of onset position (marked with a horizontal red arrow). The inset shows a logarithmic representation of the same data for lower photon energies. In this representation, the features $T_{1}-T_{3}$ can be seen more clearly. The estimated detection limit for SSPC measurements is shown as a grey area in the inset and equals a trap concentration of around $1 \times 10^{13} \mathrm{~cm}^{-3}$ [estimated using Eq. (3)]. in all samples after $\mathrm{He}$ implantation conducted at $120 \mathrm{~K}$. The features labeled $T_{4}$ and $T_{6}$ exhibit an apparent shift of their onset position after He implantation (marked with horizontal red arrows in Fig. 4). Furthermore, $T_{4}$ shows an increase in amplitude, while the apparent amplitude of $T_{6}$ decreases. There seems to be no significant impact of the He implantation on $T_{5}$. Finally, the influence of He implantation on the signature labeled $T_{2}$ is challenging to determine due to its low concentration and sporadic presence.

In Fig. 5(a), SSPC spectra are shown that were recorded on a HVPE-grown $\mathrm{Ga}_{2} \mathrm{O}_{3}$ thin film. SSPC spectra are displayed for the as-received thin film as well as for the thin film after implantation with He ions with different fluences at $120 \mathrm{~K}$. The sample was subjected to two subsequent $\mathrm{He}$ implantations, and hence two different accumulated fluences $\Phi_{\text {tot }}$. Derivative SSPC spectra are displayed for comparison in the top panel of Fig. 5(a). The derivative SSPC spectra suggest the presence of several overlapping defect signatures contributing to $T_{4}$ and $T_{6}$, which is supported by modeling the spectra (see the Supplemental Material [50]). Notably, the comparison of the derivative SSPC spectra recorded before and after He implantation at $120 \mathrm{~K}$ indicates that the subfeatures being part of $T_{4}$ and $T_{6}$ respond slightly differently to $\mathrm{He}$ implantation.

Vertical bars are displayed in Fig. 5(a) to indicate the energetic positions of the optical absorption associated with various primary intrinsic defects as predicted by computations based on hybrid functionals [see Fig. 2(b)]. Indeed, the prediction for the optical absorption related to $\mathrm{Ga}_{\mathrm{i}}^{(+/+2)}$ and $V_{\mathrm{GaI}}^{(-3 /-2)}$ overlaps with $T_{3}$, while the optical absorption related to $\mathrm{O}_{\mathrm{i}}^{(-2 /-)}, V_{\mathrm{O} K}^{(0 /+)}(K=\mathrm{I}, \mathrm{II}, \mathrm{III})$, and $V_{\mathrm{GaII}}^{(-3 /-2)}$ overlaps with $T_{4}$. Note, however, that the onset of optical absorption will depend on the absolute value of the absorption cross section, 
(a)

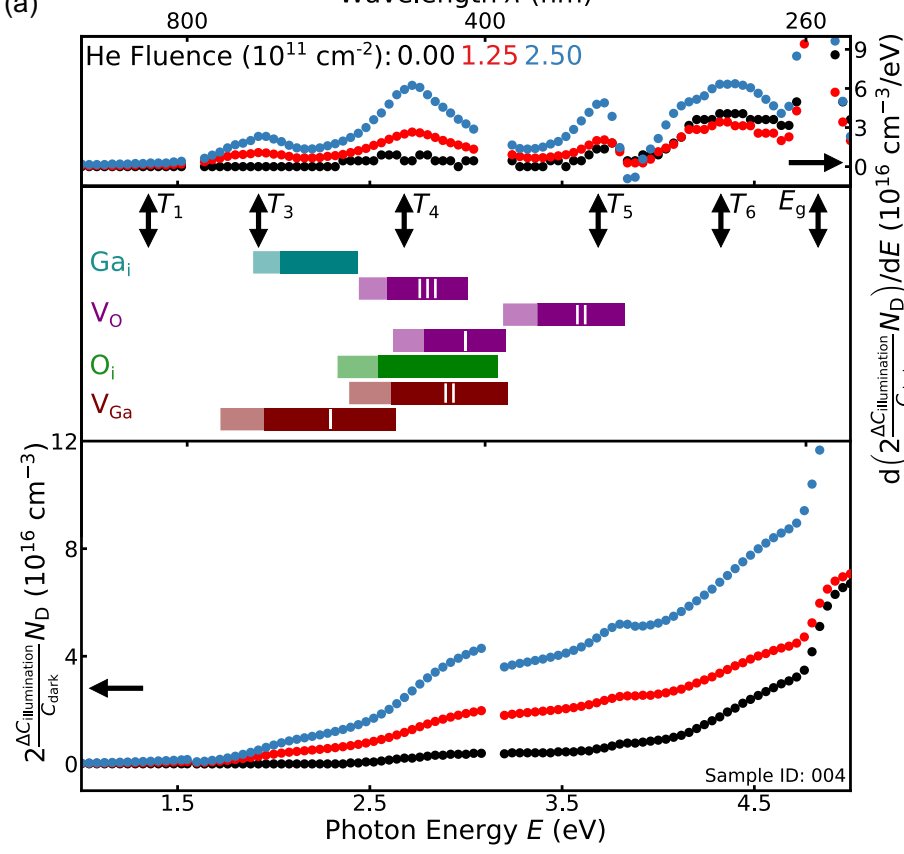

(b)

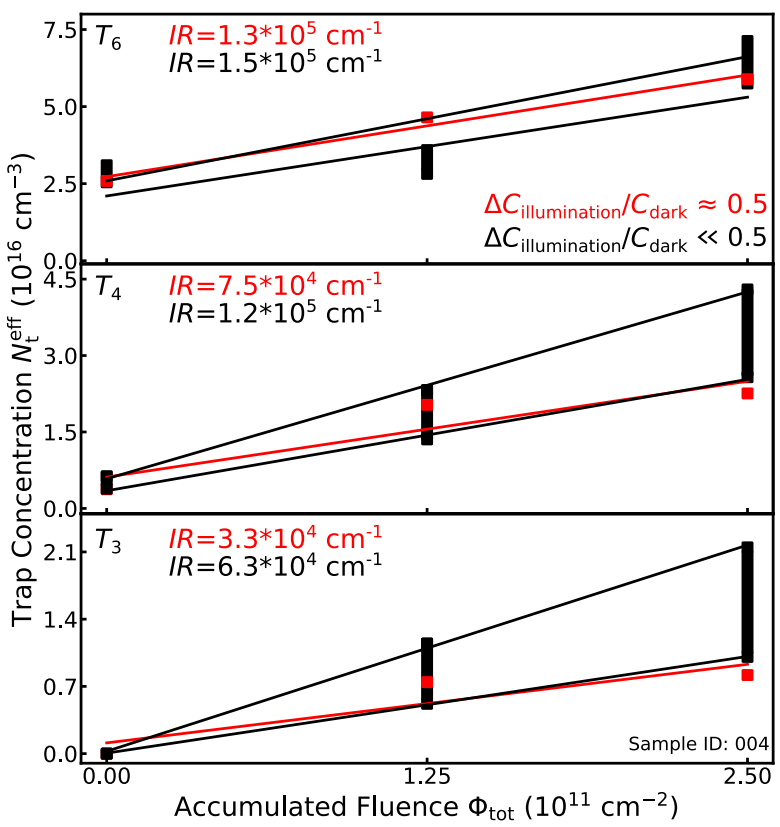

FIG. 5. (a) Results of SSPC measurements recorded on a HVPE-grown $\mathrm{Ga}_{2} \mathrm{O}_{3}$ thin film before and after subsequent implantations with $\mathrm{He}$ ions at $120 \mathrm{~K}$. The results are displayed as derivative SSPC spectra (upper panel) and as conventional SSPC spectra (lower panel). The legend states the accumulated implantation fluence $\Phi_{\text {tot }}$ for the corresponding data set. The onset positions observed in the SSPC spectra are marked with black vertical arrows. The defect signature labeled $T_{2}$ (see Fig. 4) was not detected in this sample. From the width and shape of the derivative SSPC spectra, it can be inferred that, especially, $T_{4}$ and $T_{6}$ consist of several overlapping features. Upon He implantation, $T_{3}$ is created, while $T_{4}$ as well as $T_{6}$ change in concentration. The vertical bars indicate where computations based on hybrid functionals predict the optical absorption of the primary intrinsic defects to occur (see Fig. 2). I, II, and III denote the different configurations found for $V_{\mathrm{O}}$ and $V_{\mathrm{Ga}}$ in $\mathrm{Ga}_{2} \mathrm{O}_{3}$. (b) Plot of the effective trap concentration $N_{\mathrm{t}, \mathrm{i}}^{\text {eff }}$ derived for the features labeled $T_{3}, T_{4}$, and $T_{6}$ in dependence of the accumulated He fluence $\Phi_{\mathrm{tot}}$. $N_{\mathrm{t}, \mathrm{i}}^{\text {eff }}$ was calculated for the case of $\Delta C_{\text {illumination }} / C_{\text {dark }} \ll 0.5$ [see Eq. (3)] and $\Delta C_{\text {illumination }} / C_{\text {dark }} \approx 0.5$ (see the Supplemental Material [50]). For $\Delta C_{\text {illumination }} / C_{\text {dark }} \ll 0.5$, the corresponding data points cover values computed for the case of neglecting the $\lambda$-correction as well as for assuming a Franck-Condon shift $d_{\mathrm{FC}}$ of $1 \mathrm{eV}$ (see the Supplemental Material [50]). The effective trap concentrations $N_{\mathrm{t}, \mathrm{i}}^{\text {eff }}$ associated with $T_{6}, T_{4}$, and $T_{3}$ display a linear dependence with accumulated He fluence $\Phi_{\text {tot }}$. The introduction rates IR were derived from a linear fit and are displayed in the plot.

the light intensity, as well as the competition between electron and hole processes [45,72].

From the data shown in Fig. 5(a), a plot can be constructed displaying the effective trap concentration $N_{\mathrm{t}, \mathrm{i}}^{\mathrm{eff}}$ of the individual defect levels and their dependence on $\Phi_{\text {tot }}$. The corresponding results are shown in Fig. 5(b). A linear relationship between $\Phi_{\text {tot }}$ and $N_{\mathrm{t}, \mathrm{i}}^{\text {eff }}$ is seen for the signatures $T_{3}$, $T_{4}$, and $T_{6}$. However, for $T_{6}$, a linear relationship is only seen if one assumes $\Delta C_{\text {illumination }} / C_{\text {dark }} \approx 0.5$. This is generally a better assumption than assuming $\Delta C_{\text {illumination }} / C_{\text {dark }} \ll 0.5$ for the junctions studied here. $T_{1}$ was not present in this sample. The fact that optically induced emission of electrons as well as holes is relevant for $T_{5}$ makes the determination of $N_{\mathrm{t}, \mathrm{i}}^{\mathrm{eff}}$ ambiguous, and hence the corresponding results are not shown here.

The introduction rates IR were derived from a linear fit to $N_{\mathrm{t}, \mathrm{i}}^{\text {eff }}$ versus $\Phi_{\text {tot }}$. The following introduction rates were determined for $\Delta C_{\text {illumination }} / C_{\text {dark }} \ll 0.5$ (average for the cases of neglecting the $\lambda$-correction as well as assuming a Franck-Condon shift of up to $1 \mathrm{eV}$ ): $6.3 \times 10^{4} \mathrm{~cm}^{-1}$ $\left(T_{3}\right), \quad 1.2 \times 10^{5} \mathrm{~cm}^{-1}\left(T_{4}\right)$, and $1.5 \times 10^{5} \mathrm{~cm}^{-1}\left(T_{6}\right)$. For $\Delta C_{\text {illumination }} / C_{\text {dark }} \approx 0.5$ the following introduction rates IR were computed: $3.3 \times 10^{4} \mathrm{~cm}^{-1}\left(T_{3}\right), 7.5 \times 10^{4} \mathrm{~cm}^{-1}\left(T_{4}\right)$, and $1.3 \times 10^{5} \mathrm{~cm}^{-1}\left(T_{6}\right)$. Notably, the derived IR do not differ significantly whether we assume $\Delta C_{\text {illumination }} / C_{\text {dark }} \ll 0.5$ or $\Delta C_{\text {illumination }} / C_{\text {dark }} \approx 0.5$. It should be noted that these introduction rates represent a lower bound for the actual introduction rates due to the fact that $N_{\mathrm{t}, \mathrm{i}}^{\mathrm{eff}}$ is always a lower bound for the actual trap concentration $N_{\mathrm{t}, \mathrm{i}}[44,45]$. The so-called survival rate can be computed when comparing experimental IR to the expected IR for $V_{\mathrm{Ga}}$ and $V_{\mathrm{O}}$, which was estimated to be $1 \times 10^{6} \mathrm{~cm}^{-1}$ using SRIM. Using this value, we obtain the following survival rates: $0.03-0.06\left(T_{3}\right), 0.08-0.12\left(T_{4}\right)$, and $0.13-0.15\left(T_{6}\right)$ defects per vacancy created.

Figure 6 shows SSPC spectra as well as derivative SSPC spectra recorded on a HVPE-grown $\mathrm{Ga}_{2} \mathrm{O}_{3}$ thin film before and after implantation with $\mathrm{He}$ ions at $120 \mathrm{~K}$. Additionally, results are shown for the sample after He implantation and subsequent exposure to $300 \mathrm{~K}$. The same signatures as seen in Fig. 4 were present in the corresponding $\mathrm{Ga}_{2} \mathrm{O}_{3}$ thin film before He implantation, with the exception of $T_{2}$. After He implantation, $T_{3}$ and $T_{1}$ are detected, $T_{4}$ clearly increases in concentration, while $T_{6}$ apparently decreases in concentration. After exposing the sample to $300 \mathrm{~K}$, a significant decrease in the concentration of $T_{3}$ and $T_{4}$ can be seen, while $T_{6}$ seems to increase. The signature labeled $T_{1}$ is not affected by the exposure to $300 \mathrm{~K}$. Note that these trends were seen in various samples exposed to similar implantation and annealing 


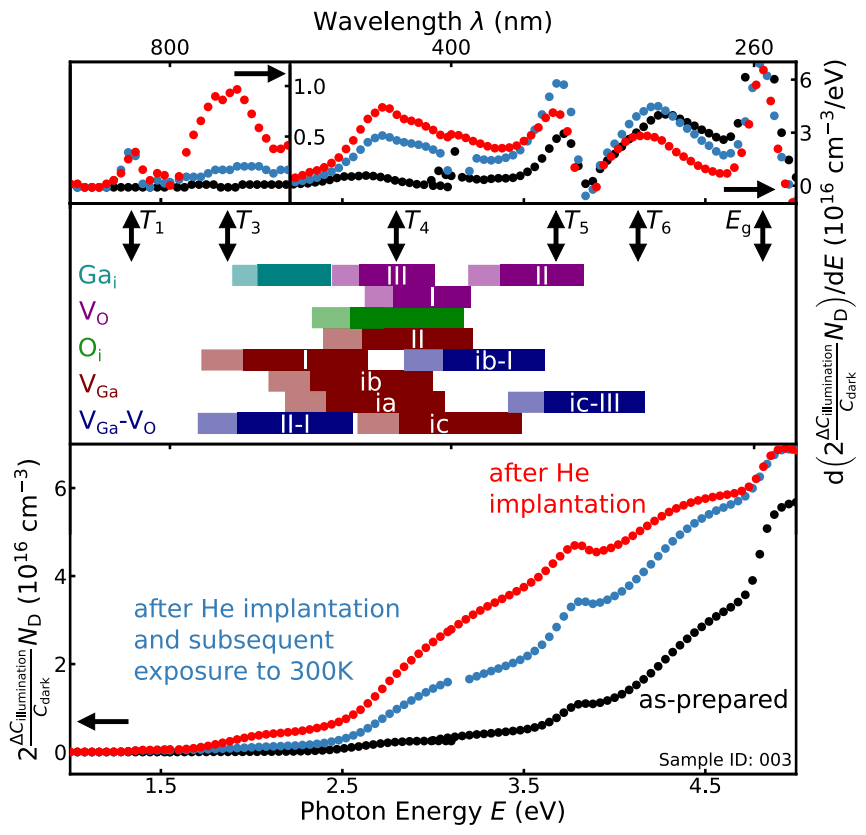

FIG. 6. SSPC spectra recorded on a HVPE-grown $\mathrm{Ga}_{2} \mathrm{O}_{3}$ thin film before and after $\mathrm{He}$ implantation at $120 \mathrm{~K}$ with a fluence of $1.25 \times 10^{11} \mathrm{~cm}^{-2}$ as well as after a subsequent annealing at $300 \mathrm{~K}$ for $\sim 5 \mathrm{~min}$. The results are displayed as derivative SSPC spectra (upper panel) and as conventional SSPC spectra (lower panel). The same signatures as seen in Fig. 4 were present in the corresponding $\mathrm{Ga}_{2} \mathrm{O}_{3}$ thin film before He implantation, with the exception of $T_{2}$. After $\mathrm{He}$ implantation, new features labeled $T_{1}$ and $T_{3}$ are observed, while the signatures $T_{4}$ and $T_{6}$ change in amplitude. Upon annealing, $T_{3}$ and $T_{4}$ decrease in amplitude, while $T_{6}$ increases in signal. $T_{1}$ seems not to be affected by the heat treatment. A panel is also shown with bars representing the computational prediction of optical signatures related to the absorption associated with various primary intrinsic defects as well as divacancy complexes. Results are also shown for the relaxed configurations of $V_{\mathrm{Ga}}$.

conditions (not shown). Figure 6 also shows bars representing the expected position of optical signatures related to the absorption associated with primary intrinsic defects as well as some divacancy complexes. Here, results are also shown for the relaxed configurations of $V_{\mathrm{Ga}}$.

\section{DISCUSSION}

On-line SSPC measurements combined with He implantation at cryogenic temperatures $(120 \mathrm{~K})$ offer a unique possibility to study the electronic states associated with optically and electrically active primary intrinsic defects, since the diffusion of defects is suppressed. In addition, the migration and/or passivation of defect species mobile at or below room temperature can be studied by subsequent heat treatments, potentially revealing further information about the observed defects. Moreover, we explicitly calculated optical absorption spectra related to the primary intrinsic defects in order to relate signatures seen in SSPC spectra to specific intrinsic defects.

SSPC spectra display several features in as-received HVPE-grown $\mathrm{Ga}_{2} \mathrm{O}_{3}$ thin films as well as features that are only visible after $\mathrm{He}$ implantation at $120 \mathrm{~K}$. The features labeled $T_{1}, T_{2}, T_{3}, T_{4}$, and $T_{6}$ are steplike, and hence indicate optically induced emission of electrons from trap levels inside the band gap to the conduction band. The feature labeled $T_{5}$, on the other hand, is peaklike, and hence clearly displays characteristics of processes related to the optical emission of electrons and holes from a trap level to the conduction band and valence band, respectively $[44,45]$. However, the electron and hole emission related to $T_{5}$ does not have to originate from the same trap. Our results are in accordance with previously reported results, where defect signatures with an onset for optical absorption at around 2.2, 3.2, and $4.4 \mathrm{eV}$ have been measured by SSPC or related techniques [23,24,26,26,27].

Starting with $T_{1}$, it is only generated in relatively low concentrations upon He implantation compared to the introduction of the other levels responding to He implantation. In addition, not all samples in the present study display the introduction of $T_{1}$ [see Fig. 5(a)]. Interestingly, Farzana et al. also observed the introduction of a new defect signature with an onset at around $1.3 \mathrm{eV}$ in SSPC measurements after neutron irradiation [28]. Furthermore, Polyakov et al. revealed a level with approximately the same onset energy as $T_{1}$ that responded to proton irradiation using $\mathrm{CV}$ measurements under illumination $[23,24]$. The onsets for optical absorption estimated from hybrid-functional calculations corroborate that no primary intrinsic defect exhibits an onset for optical absorption as low as the one observed for $T_{1}$. Hence, we propose that $T_{1}$ does not arise from a primary intrinsic defect, but rather from a defect complex or an impurity. Further, $T_{1}$ is not affected by exposure of the sample to $300 \mathrm{~K}$ after $\mathrm{He}$ implantation at $120 \mathrm{~K}$, suggesting that $T_{1}$ is related to a defect that is not mobile at or below room temperature.

$T_{5}$ does not seem to exhibit a pronounced response to $\mathrm{He}$ implantation. The same applies to the signature labeled $T_{2}$, which is not detected for all samples investigated. This lack of response to He implantation indicates that no primary intrinsic defects are involved, but rather there is an impurity or a defect complex.

For $T_{3}, T_{4}$, and $T_{6}$, an approximately linear relationship is seen for their effective trap concentrations in dependence on the accumulated fluence of implanted He ions. Comparing the measured introduction rates with SRIM simulations, the introduced trap concentrations corresponding to $T_{3}, T_{4}$, and $T_{6}$ equal about $4 \%, 10 \%$, and $14 \%$ of what is expected for Frenkel-pair generation, respectively. Thus, the IR values for $T_{3}, T_{4}$, and $T_{6}$ are close to those expected for primary intrinsic defects.

After implanting $\mathrm{He}$ ions at $120 \mathrm{~K}$, one can expect that $\mathrm{Ga}_{\mathrm{i}}, V_{\mathrm{Ga} J}(J=\mathrm{I}, \mathrm{II})$, and $V_{\mathrm{O} K}(K=\mathrm{I}$, II, III $)$ will be present, while it is likely that $\mathrm{O}_{\mathrm{i}}$ will already have migrated out of the implanted volume and/or formed complexes. Notably, the transformation of $V_{\mathrm{Ga} J}(J=\mathrm{I}, \mathrm{II})$ into $V_{\mathrm{Ga}}^{\mathrm{i} M}(M=a, c, b)$ is also not expected to occur at $120 \mathrm{~K}$. Even if some defect migration or transformation occurs at $120 \mathrm{~K}$, one can at least expect complex formation to be substantially suppressed.

Comparing the onset of $T_{3}$ with the optical absorption of the primary intrinsic defects obtained from the hybridfunctionals calculations, it is evident that the expected onsets for optical absorption computed for $\mathrm{Ga}_{\mathrm{i}}^{(+/+2)}$ and $V_{\mathrm{GaI}}^{(-3 /-2)}$ are close to the position of $T_{3}$. Importantly, the optical absorption 
expected for $V_{\mathrm{GaII}}, V_{\mathrm{O} K}(K=\mathrm{I}, \mathrm{II}, \mathrm{III})$, and $\mathrm{O}_{\mathrm{i}}$ is located at higher photon energies. The optical signatures related to $V_{\mathrm{GaII}}^{(-3 /-2)}, V_{\mathrm{OK}}^{(0 /+)}(K=\mathrm{I}, \mathrm{II}, \mathrm{III})$, and $\mathrm{O}_{\mathrm{i}}^{(-2 /-)}$ are, however, close to $T_{4}$. Thus, $\mathrm{Ga}_{\mathrm{i}}$ and $V_{\mathrm{GaI}}$ are potential candidates for $T_{3}$, whereas $V_{\mathrm{GaII}}$ and $V_{\mathrm{OK}}(K=\mathrm{I}$, II, III $)$ are proposed as candidates for $T_{4}$.

However, some of the primary intrinsic defects exhibit several optical charge-state transition levels in the band gap of $\mathrm{Ga}_{2} \mathrm{O}_{3}$ (see the Supplemental Material [50]). In particular, optical transitions related to $V_{\mathrm{Ga} J}^{(-2 /-1)}$ and $V_{\mathrm{Ga} J}^{(-1 / 0)}(J=\mathrm{I}$, II) may also contribute to $T_{4}$. However, the concentration of the defects associated with $T_{4}$ is significantly higher than the defect concentration related to $T_{3}$, and hence some defects contributing to $T_{4}$ are not likely to contribute to $T_{3}$.

The exposure of samples implanted with $\mathrm{He}$ at cryogenic temperatures to room temperature can further aid the assignment of defect signatures seen in SSPC measurements to specific intrinsic defects. The observed decrease in concentration for $T_{3}$ and $T_{4}$ upon room-temperature exposure may be explained by annihilation reactions of $\mathrm{Ga}_{\mathrm{i}}$ and $V_{\mathrm{Ga}}$ defects. Such annihilation reactions lead to a decrease in $\mathrm{Ga}_{i}$ as well as $V_{\mathrm{Ga} J}(J=\mathrm{I}, \mathrm{II})$, and hence to a decrease of $T_{3}$ and $T_{4}$. A transformation of $V_{\mathrm{Ga} J}(J=\mathrm{I}, \mathrm{II})$ into $V_{\mathrm{Ga}}^{\mathrm{i} M}(M=a, c, b)$ should, in contrast, lead to a decrease in the concentration associated with $T_{3}$ and an increase in the concentration associated with $T_{4}$, taking into account that the transitions related to $V_{\mathrm{Ga}}^{\mathrm{i} M}(M=a, c, b)$ are predicted to be close to $T_{4}$. However, it is not possible to rule out that both processes, annihilation of $\mathrm{Ga}_{\mathrm{i}}$ with $V_{\mathrm{Ga}}$ as well as transformation of $V_{\mathrm{Ga}}$, may contribute to the observed changes in photocapacitance.

Finally, $T_{6}$ exhibits an increase in concentration with increasing fluence of $\mathrm{He}$ implanted at $120 \mathrm{~K}$, in addition to a distinct increase in concentration after exposure of the samples to room temperature. As mentioned above, several of the intrinsic defects also exhibit charge states in the lower part of the band gap (see the Supplemental Material [50]), and might contribute to $T_{6}$. However, the increase in $T_{6}$, while $T_{3}$ and $T_{4}$ decrease, suggests different origins for $T_{6}$ compared to $T_{3}$ as well as $T_{4}$. Hence, it is tempting to propose that $T_{6}$ involves a defect complex, rather than a primary intrinsic defect, and divacancies may also be potential candidates for $T_{6}$, although the calculated absorption spectra do not result in an adequate fit to the measured position of $T_{6}$. Thus, further investigations are required to shed light on the origin of the $T_{6}$ level.

\section{SUMMARY AND CONCLUSION}

Using on-line SSPC measurements, we were able to observe optical transitions related to defects in HVPE-grown $\mathrm{Ga}_{2} \mathrm{O}_{3}$ thin films with onset energies at 1.3, 1.7, 1.9, 2.6, 3.7 , and $4.2 \mathrm{eV}$. The levels were labeled $T_{1}-T_{6}$ in ascending order of onset energy. $T_{2}, T_{4}, T_{5}$, and $T_{6}$ are observed in all as-received HVPE-grown $\mathrm{Ga}_{2} \mathrm{O}_{3}$ thin films, whereby $T_{2}$ is only observed sporadically. $T_{1}$ and $T_{3}$ are only detected after He implantation at $120 \mathrm{~K} . T_{4}$ and $T_{6}$ also respond to $\mathrm{He}$ implantation. The introduction rates for $T_{3}, T_{4}$, as well as $T_{6}$ indicate their relation to primary intrinsic defects. We also calculated migration barriers for $\mathrm{Ga}_{i}$, and we used reported migration barriers for the vacancy defects [14] and $\mathrm{O}_{i}$ [15]. Thus, we expect $\mathrm{O}_{\mathrm{i}}$ to be mobile at temperatures below $120 \mathrm{~K}$, and hence to not be present in our samples after He implantation at $120 \mathrm{~K}$. $V_{\mathrm{OK}}(K=\mathrm{I}, \mathrm{II}, \mathrm{III})$ are not expected to be mobile below room temperature, while $\mathrm{Ga}_{\mathrm{i}}$ should be immobile at $120 \mathrm{~K}$, but be mobile at or below room temperature. $V_{\mathrm{Ga} J}$ ( $J=$ I, II) might be mobile just below room temperature, and it is predicted to transform into relaxed configurations that are stable at room temperature $[14,19]$. We performed hybrid-functional calculations in order to predict the optical absorption cross-section spectra for various primary intrinsic defects. Thus, we tentatively propose $T_{3}$ to be associated with $\mathrm{Ga}_{\mathrm{i}}^{(+/+2)}$ and/or $V_{\mathrm{GaI}}^{(-3 /-2)}$, whereas $T_{4}$ is suggested to be related to $V_{\mathrm{O} K}^{(0 /+)}(K=\mathrm{I}, \mathrm{II}, \mathrm{III})$ and/or $V_{\mathrm{GaII}}^{(-3 /-2)}$, although an overlap with other charge-state transitions, such as $V_{\mathrm{Ga} J}^{(-2 /-1)}$ and $V_{\mathrm{Ga} J}^{(-1 / 0)}(J=\mathrm{I}, \mathrm{II})$, cannot be excluded.

Further insights were gained by studying the kinetics of the defects created upon He implantation at $120 \mathrm{~K}$. We found that $T_{3}, T_{4}$, and $T_{6}$ change in concentration upon exposing samples to room temperature after $\mathrm{He}$ implantation at $120 \mathrm{~K}$. $T_{3}$ and $T_{4}$ decrease in concentration upon such annealing, while $T_{6}$ increases in concentration. The kinetics observed for $T_{3}$ and $T_{4}$ further support the proposed assignments of the corresponding defect signatures to $\mathrm{Ga}_{\mathrm{i}}$ and $V_{\mathrm{Ga}}$ due to $\mathrm{Ga}_{\mathrm{i}}$ being mobile already at or below room temperature, and hence annihilation of $\mathrm{Ga}_{\mathrm{i}}$ and $V_{\mathrm{Ga}}$ to be a likely mechanism. For $T_{6}$, complex formation or passivation involving other defects are proposed to be involved.

\section{ACKNOWLEDGMENTS}

Financial support is acknowledged from the Research Council of Norway through the FUNDAMENT project (Project No. 251131), the Norwegian Micro- and NanoFabrication Facility (NorFab, Project No. 245963), and the Faculty of Mathematics and Natural Sciences at the University of Oslo via the strategic research initiative FOXHOUND. This work was partially performed under the auspices of the US DOE by Lawrence Livermore National Laboratory under Contract No. DE-AC52-07NA27344, and supported by the Critical Materials Institute, an Energy Innovation Hub funded by the US DOE, Office of Energy Efficiency and Renewable Energy, Advanced Manufacturing Office. We wish to thank Audrius Alkauskas for fruitful collaborations and discussions. We further thank Ole Myren Røhne and Ole Dorholt for wirebonding of our samples, and Josef Gert Åsheim Ellingsen for proofreading the manuscript.
[1] M. Higashiwaki, K. Sasaki, H. Murakami, Y. Kumagai, A. Koukitu, A. Kuramata, T. Masui, and S. Yamakoshi, Semicond. Sci. Technol. 31, 034001 (2016).
[2] S. Nakagomi, T. Momo, S. Takahashi, and Y. Kokubun, Appl. Phys. Lett. 103, 072105 (2013). 
[3] S. Nakagomi, T. Sato, Y. Takahashi, and Y. Kokubun, Sens. Actuators A 232, 208 (2015).

[4] M. A. Mastro, A. Kuramata, J. Calkins, J. Kim, F. Ren, and S. Pearton, ECS J. Solid State Sci. Technol. 6, P356 (2017).

[5] S. Ahn, Y.-H. Lin, F. Ren, S. Oh, Y. Jung, G. Yang, J. Kim, M. A. Mastro, J. K. Hite, C. R. Eddy Jr. et al., J. Vac. Sci. Technol. B 34, 041213 (2016).

[6] J. F. McGlone, Z. Xia, Y. Zhang, C. Joishi, S. Lodha, S. Rajan, S. A. Ringel, and A. R. Arehart, IEEE Electron Device Lett. 39, 1042 (2018).

[7] J. F. McGlone, Z. Xia, C. Joishi, S. Lodha, S. Rajan, S. Ringel, and A. R. Arehart, Appl. Phys. Lett. 115, 153501 (2019).

[8] J. Lee, S. Ganguli, A. K. Roy, and S. C. Badescu, J. Chem. Phys. 150, 174706 (2019).

[9] T. Zacherle, P. C. Schmidt, and M. Martin, Phys. Rev. B 87, 235206 (2013).

[10] S. Lany, APL Mater. 6, 046103 (2018).

[11] J. R. Ritter, J. Huso, P. T. Dickens, J. B. Varley, K. G. Lynn, and M. D. McCluskey, Appl. Phys. Lett. 113, 052101 (2018).

[12] J. L. Lyons, Semicond. Sci. Technol. 33, 05LT02 (2018).

[13] A. Kyrtsos, M. Matsubara, and E. Bellotti, Appl. Phys. Lett. 112, 032108 (2018).

[14] A. Kyrtsos, M. Matsubara, and E. Bellotti, Phys. Rev. B 95, 245202 (2017).

[15] M. Ingebrigtsen, A. Y. Kuznetsov, B. Svensson, G. Alfieri, A. Mihaila, U. Badstübner, A. Perron, L. Vines, and J. Varley, APL Mater. 7, 022510 (2019).

[16] J. Varley, J. Weber, A. Janotti, and C. Van de Walle, Appl. Phys. Lett. 97, 142106 (2010).

[17] P. Deák, Q. Duy Ho, F. Seemann, B. Aradi, M. Lorke, and T. Frauenheim, Phys. Rev. B 95, 075208 (2017).

[18] H. J. von Bardeleben, S. Zhou, U. Gerstmann, D. Skachkov, W. R. Lambrecht, Q. D. Ho, and P. Deák, APL Mater. 7, 022521 (2019).

[19] J. B. Varley, H. Peelaers, A. Janotti, and C. G. Van de Walle, J. Phys.: Condens. Matter 23, 334212 (2011).

[20] K. Irmscher, Z. Galazka, M. Pietsch, R. Uecker, and R. Fornari, J. Appl. Phys. 110, 063720 (2011).

[21] M. E. Ingebrigtsen, J. Varley, A. Y. Kuznetsov, B. G. Svensson, G. Alfieri, A. Mihaila, U. Badstübner, and L. Vines, Appl. Phys. Lett. 112, 042104 (2018).

[22] A. Polyakov, N. Smirnov, I. Shchemerov, E. Yakimov, J. Yang, F. Ren, G. Yang, J. Kim, A. Kuramata, and S. Pearton, Appl. Phys. Lett. 112, 032107 (2018).

[23] A. Polyakov, N. Smirnov, I. Shchemerov, E. Yakimov, S. Pearton, C. Fares, J. Yang, F. Ren, J. Kim, P. Lagov et al., Appl. Phys. Lett. 113, 092102 (2018).

[24] A. Polyakov, N. Smirnov, I. Shchemerov, S. Pearton, F. Ren, A. Chernykh, P. Lagov, and T. V. Kulevoy, APL Mater. 6, 096102 (2018).

[25] A. Polyakov, N. Smirnov, I. Shchemerov, D. Gogova, S. Tarelkin, and S. Pearton, J. Appl. Phys. 123, 115702 (2018).

[26] E. Farzana, E. Ahmadi, J. S. Speck, A. R. Arehart, and S. A. Ringel, J. Appl. Phys. 123, 161410 (2018).

[27] Z. Zhang, E. Farzana, A. Arehart, and S. Ringel, Appl. Phys. Lett. 108, 052105 (2016).

[28] E. Farzana, M. F. Chaiken, T. E. Blue, A. R. Arehart, and S. A. Ringel, APL Mater. 7, 022502 (2019).
[29] A. Polyakov, N. Smirnov, I. Shchemerov, S. Pearton, F. Ren, A. Chernykh, and A. Kochkova, Appl. Phys. Lett. 113, 142102 (2018).

[30] Y. Nakano, ECS J. Solid State Sci. Technol. 6, P615 (2017).

[31] H. Gao, S. Muralidharan, N. Pronin, M. R. Karim, S. M. White, T. Asel, G. Foster, S. Krishnamoorthy, S. Rajan, L. R. Cao et al., Appl. Phys. Lett. 112, 242102 (2018).

[32] S.-S. Huang, R. Lopez, S. Paul, A. T. Neal, S. Mou, M.-P. Houng, and J. V. Li, Jpn. J. Appl. Phys. 57, 091101 (2018).

[33] A. T. Neal, S. Mou, S. Rafique, H. Zhao, E. Ahmadi, J. S. Speck, K. T. Stevens, J. D. Blevins, D. B. Thomson, N. Moser et al., Appl. Phys. Lett. 113, 062101 (2018).

[34] L. Binet and D. Gourier, Appl. Phys. Lett. 77, 1138 (2000).

[35] G. Schmitz, P. Gassmann, and R. Franchy, J. Appl. Phys. 83, 2533 (1998).

[36] J. Kim, S. J. Pearton, C. Fares, J. Yang, F. Ren, S. Kim, and A. Y. Polyakov, J. Mater. Chem. C 7, 10 (2019).

[37] L. Vines, C. Bhoodoo, H. Von Wenckstern, and M. Grundmann, J. Phys.: Condens. Matter 30, 025502 (2017).

[38] M. A. Blanco, M. B. Sahariah, H. Jiang, A. Costales, and R. Pandey, Phys. Rev. B 72, 184103 (2005).

[39] P. Weiser, M. Stavola, W. B. Fowler, Y. Qin, and S. Pearton, Appl. Phys. Lett. 112, 232104 (2018).

[40] C. Bhoodoo, A. Hupfer, L. Vines, E. V. Monakhov, and B. G. Svensson, Phys. Rev. B 94, 205204 (2016).

[41] A. Hupfer, C. Bhoodoo, L. Vines, and B. G. Svensson, J. Appl. Phys. 119, 181506 (2016).

[42] J. F. Ziegler, M. D. Ziegler, and J. P. Biersack, Nucl. Instrum. Methods Phys. Res. Sec. B 268, 1818 (2010).

[43] M. Passlack, N. E. J. Hunt, E. F. Schubert, G. J. Zydzik, M. Hong, J. P. Mannaerts, R. L. Opila, and R. J. Fischer, Appl. Phys. Lett. 64, 2715 (1994).

[44] P. Blood and J. Orton, The Electrical Characterization of Semiconductors: Majority Carriers and Electron States, Techniques of Physics No. 2 (Academic, San Diego, CA, 1992).

[45] A. Chantre, G. Vincent, and D. Bois, Phys. Rev. B 23, 5335 (1981).

[46] A. White, P. Dean, and P. Porteous, J. Appl. Phys. 47, 3230 (1976).

[47] T. P. Weiss, A. Redinger, D. Regesch, M. Mousel, and S. Siebentritt, IEEE J. Photovolt. 4, 1665 (2014).

[48] C. G. Van de Walle and J. Neugebauer, J. Appl. Phys. 95, 3851 (2004).

[49] A. Alkauskas, M. D. McCluskey, and C. G. Van de Walle, J. Appl. Phys. 119, 181101 (2016).

[50] See Supplemental Material at http://link.aps.org/supplemental/ 10.1103/PhysRevMaterials.4.074605 for more information regarding the extraction of trap concentrations from steady-state photocapacitance measurements, and for more information on first-principles calculations of structures, optical charge-state transition levels, and migration barriers of primary intrinsic defects in $\beta-\mathrm{Ga}_{2} \mathrm{O}_{3}$, including Refs. [73-76].

[51] P. E. Blöchl, Phys. Rev. B 50, 17953 (1994).

[52] G. Kresse and D. Joubert, Phys. Rev. B 59, 1758 (1999).

[53] A. V. Krukau, O. A. Vydrov, A. F. Izmaylov, and G. E. Scuseria, J. Chem. Phys. 125, 224106 (2006).

[54] G. Kresse and J. Furthmüller, Phys. Rev. B 54, 11169 (1996).

[55] C. Janowitz, V. Scherer, M. Mohamed, A. Krapf, H. Dwelk, R. Manzke, Z. Galazka, R. Uecker, K. Irmscher, R. Fornari et al., New J. Phys. 13, 085014 (2011). 
[56] T. Onuma, S. Saito, K. Sasaki, K. Goto, T. Masui, T. Yamaguchi, T. Honda, A. Kuramata, and M. Higashiwaki, Appl. Phys. Lett. 108, 101904 (2016).

[57] C. Freysoldt, B. Grabowski, T. Hickel, J. Neugebauer, G. Kresse, A. Janotti, and C. G. Van de Walle, Rev. Mod. Phys. 86, 253 (2014).

[58] Y. Kumagai and F. Oba, Phys. Rev. B 89, 195205 (2014).

[59] C. Freysoldt, J. Neugebauer, and C. G. Van de Walle, Phys. Rev. Lett. 102, 016402 (2009).

[60] T. Gake, Y. Kumagai, C. Freysoldt, and F. Oba, Phys. Rev. B 101, 020102(R) (2020).

[61] A. Kopylov and A. Pikhtin, Sov. Phys. Solid State 16, 1200 (1975).

[62] T. Onuma, S. Saito, K. Sasaki, T. Masui, T. Yamaguchi, T. Honda, and M. Higashiwaki, Jpn. J. Appl. Phys. 54, 112601 (2015).

[63] H. Peelaers and C. G. Van de Walle, Appl. Phys. Lett. 111, 182104 (2017).

[64] J. L. Lyons, A. Alkauskas, A. Janotti, and C. G. Van de Walle, Phys. Status Solidi B 252, 900 (2015).

[65] G. Lucovsky, Solid State Commun. 3, 299 (1965).

[66] G. Henkelman, B. P. Uberuaga, and H. Jónsson, J. Chem. Phys. 113, 9901 (2000).
[67] J. P. Perdew, A. Ruzsinszky, G. I. Csonka, O. A. Vydrov, G. E. Scuseria, L. A. Constantin, X. Zhou, and K. Burke, Phys. Rev. Lett. 100, 136406 (2008).

[68] J. Varley, A. Janotti, C. Franchini, and C. G. Van de Walle, Phys. Rev. B 85, 081109(R) (2012).

[69] B. E. Kananen, N. C. Giles, L. E. Halliburton, G. K. Foundos, K. B. Chang, and K. Stevens, J. Appl. Phys. 122, 215703 (2017).

[70] K. H. Warnick, Y. Puzyrev, T. Roy, D. M. Fleetwood, R. D. Schrimpf, and S. T. Pantelides, Phys. Rev. B 84, 214109 (2011).

[71] M. Schubert, R. Korlacki, S. Knight, T. Hofmann, S. Schöche, V. Darakchieva, E. Janzén, B. Monemar, D. Gogova, Q.-T. Thieu, R. Togashi, H. Murakami, Y. Kumagai, K. Goto, A. Kuramata, S. Yamakoshi, and M. Higashiwaki, Phys. Rev. B 93, 125209 (2016).

[72] Y. Furukawa, Jpn. J. Appl. Phys. 6, 675 (1967).

[73] B. Monemar and L. Samuelson, Phys. Rev. B 18, 809 (1978).

[74] T. Takebe, J. Saraie, and H. Matsunami, J. Appl. Phys. 53, 457 (1982).

[75] R. Pässler, J. Appl. Phys. 96, 715 (2004).

[76] D. Wickramaratne, C. E. Dreyer, B. Monserrat, J.-X. Shen, J. L. Lyons, A. Alkauskas, and C. G. Van de Walle, Appl. Phys. Lett. 113, 192106 (2018). 